\title{
The Ultimate Resolution in Aberration-Corrected STEM
}

\author{
S. J. Pennycook ${ }^{1}$, A. R. Lupini ${ }^{1}$, and P. D. Nellist ${ }^{2}$ \\ ${ }^{1}$ Solid State Division, Oak Ridge National Laboratory, Oak Ridge, TN $37831-6030$ USA. \\ ${ }^{2}$ Nion Co., 1102 8th St., Kirkland, WA 98033, USA.
}

Aberration correction on the STEM offers the potential to reach the fundamental quantummechanical limit for resolution in zone-axis crystals. In free space, plane waves are good quantum mechanical stationary states to describe the propagation of an electron, but mt in a zone axis crystal. Here the electron must take on the periodicity of the crystal and it propagates as Bloch states. The fundamental resolution limit is therefore the smallest Bloch state. Figure 1 shows the first few Bloch states for $\mathrm{Si}\langle 110\rangle$, where the most localized state is the 1s state. These states are the most deeply bound in the potential well of the columns, and are typically $0.5-0.8 \AA$ in diameter dependent on the strength of the potential well. This is comparable to the probe sizes predicted after correction of aberrations.

For axial illumination the antisymmetric p-type Bloch states are not excited in a perfect crystal. In phase contrast microscopy, imaging with 1s Bloch states can be achieved by selecting a sample thickness in which the contribution of the 1s state to the exit face wave function is maximized. This occurs at a thickness of $\xi / 4$, where $\xi$ is the extinction distance. But this thickness is different for columns of different composition; at thicknesses greater than $\xi / 4$, the column reverses contrast. In Z-contrast imaging it is the detector that provides the filtering. The inner detector angle in increased until only the most localized states contribute to the intensity. The large angular integration ensures transverse incoherence, and phonon scattering ensures longitudinal incoherence. It was recognized long ago that the 1s states were the dominant contribution to the image, and their non-dispersive nature was necessary for the incoherent nature of the image.[1] However, by assuming the image intensity to be proportional to the intensity at the atom sites, the detector geometry was not included. Recently it has been shown that the detector provides more perfect Bloch state filtering than originally thought.[2] The 1s states are responsible for the image contrast even when the $2 \mathrm{~s}$ states are more highly excited. As the probe size is reduced in size, eventually, the Z-contrast image will become a direct image of the 1s Bloch states.

For EELS, there has been much discussion on delocalization, that inner shell excitation could be achieved from a point charge passing at a distance. Classical expressions for the impact parameter are velocity-dependent, but quantum mechanical predictions are not. Furthermore, use of the dipole approximation is invalid in the present context. For atomic resolution EELS, large acceptance angles are necessary and we are interested in the response at large distance. Thus we cannot expand $\mathrm{e}^{\mathrm{iq \cdot r}}$ by $1+$ iq.r. Doing so suggests significant delocalization (Fig 2) [3] but this is not seen with the full calculation. [4] In this case the ultimate resolution for a single atom is very close to the geometric size of the inner shell. Delocalization is negligible. For zone axis crystals the ultimate resolution is again the 1s Bloch state. 


\section{References:}

[1] S. J. Pennycook and D. E. Jesson, Phys Rev Lett, 64 (1990) 938, Ultramicroscopy, 37 (1991) 14.

[2] P. D. Nellist and S. J. Pennycook, Ultramicroscopy, 78 (1999) 111.

[3] D. A. Muller and J. Silcox, Ultramicroscopy, 59 (1995) 195.

[4] B. Rafferty and S. J. Pennycook, Ultramicroscopy, 78 (1999) 141.

[5] V. W. Maslen and C. J. Rossouw, Philos Mag A,49 (1984) 735.

[6] This work was supported by the USDOE under contract DEAC05-00OR22725 managed by UTBattelle, LLC.

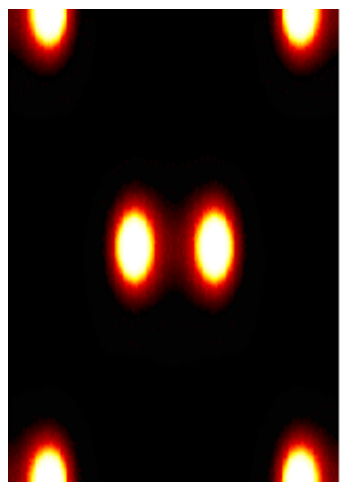

1s-like

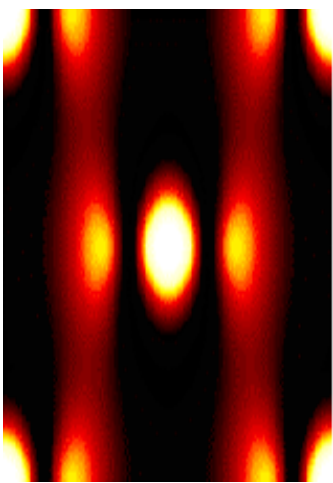

2s-like

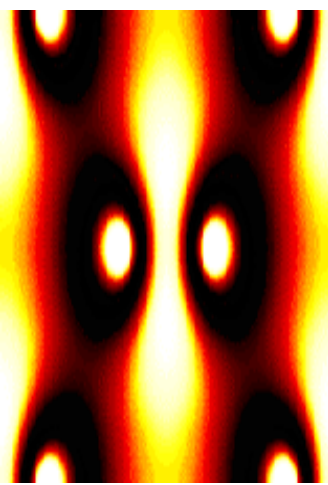

$2 p_{y}$-like

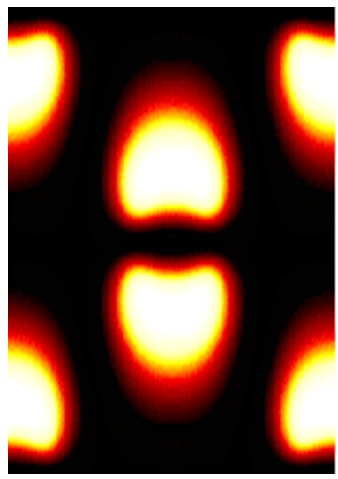

$2 p_{x}$-like

Fig. 1. Bloch state intensities for $\mathrm{Si}\langle 110\rangle$.

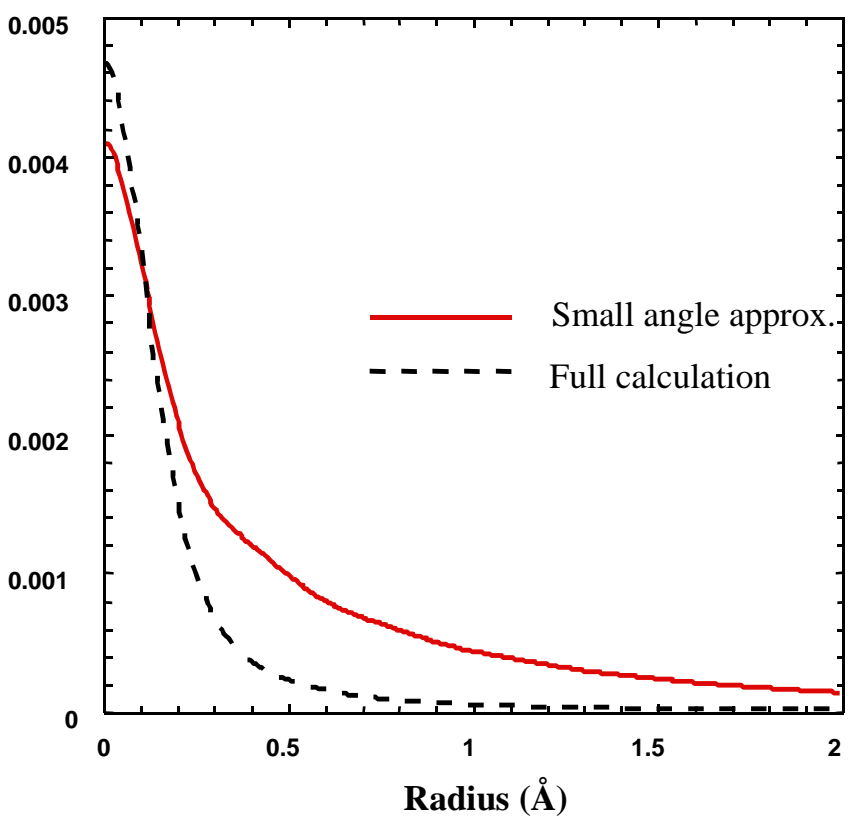

Fig. 2. Spatial distribution of EELS intensity for an infinitesimal probe comparing the dipole expansion to the full calculation.

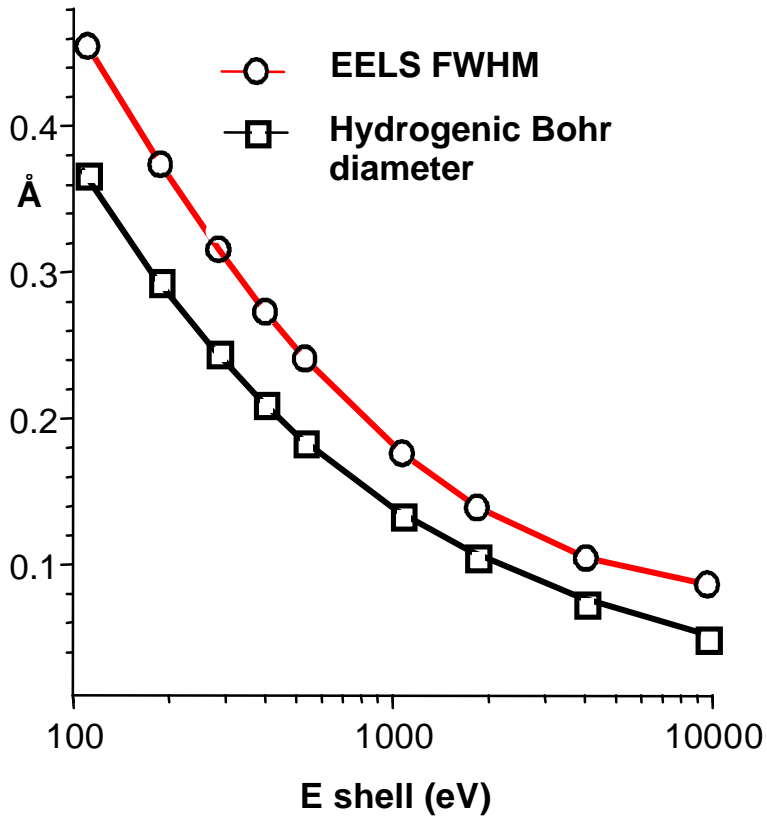

Fig. 3. Full width half maximum of the EELS object function compared to the diameter of the inner shell. Calculations use the hydrogenic model. [5] 